\title{
Restricted 2-Factor Polytopes
}

\author{
William H. Cunningham * \\ Department of Combinatorics and Optimization \\ University of Waterloo \\ Waterloo, Ontario N2L 3G1 \\ and \\ Yaoguang Wang \\ Max-Planck-Institut für Informatik \\ Im Stadtwald \\ 66123 Saarbrucken, Germany
}

February 25, 1997

\begin{abstract}
The optimal $k$-restricted 2 -factor problem consists of finding, in a complete undirected graph $K_{n}$, a minimum cost 2-factor (subgraph having degree 2 at every node) with all components having more than $k$ nodes. The problem is a relaxation of the well-known symmetric travelling salesman problem, and is equivalent to it when $\frac{n}{2} \leq k \leq n-1$. We study the $k$-restricted 2 -factor polytope. We present a large class of valid inequalities, called bipartition inequalities, and describe some of their properties; some of these results are new even for the travelling salesman polytope. For the case $k=3$, the triangle-free 2 -factor polytope, we derive a necessary and sufficient condition for such inequalities to be facet inducing.
\end{abstract}

${ }^{*}$ Research partially supported by a grant from N.S.E.R.C. of Canada 


\section{Introduction}

A 2-factor of an undirected graph $G=(V, E)$ is a spanning subgraph $H$ of $G$ that has degree 2 at each node. Equivalently, it is a set of node-disjoint circuits that include all of the nodes. Of course, a special case is a Hamiltonian circuit of $G$. Deciding whether $G$ has a Hamiltonian circuit is a well-known NP-complete problem, whereas deciding whether $G$ has a 2-factor can be done in polynomial time, due to its equivalence to problems in matching. We consider a class of problems intermediate in difficulty to these two problems. A 2-factor $H$ is $k$-restricted, (or just restricted), for $k$ an integer, if each component of $H$ has more than $k$ nodes. If $k=n-1$ (in fact, if $n / 2 \leq k \leq n-1$ ), then any restricted 2-factor is a Hamiltonian circuit. If $k=0$, then every 2-factor is a restricted 2-factor.

In fact, much is known about the complexity of determining whether a given $G$ has a restricted 2-factor. For $k=0,1,2$, matching techniques due to Edmonds can be used to answer the question efficiently. (Note that these problems are all equivalent if the graph has no loops or multiple edges, but otherwise there are differences.) For $k=3$ ("triangle-free 2-factors"), Hartvigsen [9] gave an efficient algorithm. His solution is difficult. On the other hand, for $k \geq 5$ the problem has been proved to be NP-complete by Papadimitriou; see [3]. So from the point of view of computational complexity, only the case $k=4$ remains open.

The optimal restricted 2-factor problem is, given $G=(V, E)$ and edge weights $\left(c_{e}\right.$ : $e \in E$ ), to find a maximum-weight restricted 2-factor. Of course, this optimization problem is at least as hard as the corresponding decision problem discussed above. So it is NP-hard for $k \geq 5$. In fact, Vornberger [10] has proved that the optimization problem is NP-hard for $k=4$ also. So from the complexity point of view, the only open case is $k=3$, the "optimal triangle-free 2 -factor problem". If one assumes that the graph is complete and the weight function satisfies the triangle inequality, then the optimization problem remains NP-hard for $k \geq 4$. However, under this assumption there is a polynomial-time algorithm that guarantees to find a solution of weight at most twice optimal; see Goemans and Williamson [6].

In this paper we study these problems from a polyhedral viewpoint. There is a great deal of evidence that this approach can lead to linear-programming based techniques that 
provide excellent bounds and even provably optimal solutions. An example, which has received a lot of attention, is the (symmetric) travelling salesman problem (TSP). One motivation for polyhedral results on restricted 2-factors is that they generalize results for the TSP. Another is the open case $k=3$ for which (unlike the NP-hard cases) we may hope for a complete description of the polyhedron. A third is that there seems to be a sense in which for smaller values of $k$ the polyhedron is better-behaved.

We consider mainly the "bipartition inequalities", a class of inequalities that was introduced for the TSP by Boyd and Cunningham [1]. They include well-known earlier classes like subtour elimination, comb, and clique-tree inequalities. They extend the clique-tree class by dropping the restriction to a tree structure, and by allowing some of the "teeth" to be "degenerate". There is a natural way to choose a subfamily of bipartition inequalities for each $k$, namely, by requiring teeth to have size at most $k$, and this restricted family, called " $k$-bipartition inequalities", turns out to be valid for the restricted 2 -factor problem for that $k$. We prove that for a $k$-bipartition inequality to be facet-inducing for the restricted 2-factor polytope, it must satisfy a certain connectivity condition, namely, it can have no cutset consisting of degenerate teeth. This result is new even for the TSP polytope. Moreover, for $k=3$, we obtain necessary and sufficient conditions for a $k$-bipartition inequality to be facet-inducing. The resulting class is large, but it has a nice structure. However, it is unfortunately not complete; we also show that even for $k=3$, there are facet-inducing inequalities that are not in this class.

The paper is organized as follows. Section 2 consists of some preliminaries. In Section 3 , we describe the class of bipartition inequalities and prove their validity for the $k^{-}$ restricted 2-factor polytope. In Section 4, we prove necessary conditions for a bipartition inequality to be facet inducing, and conjecture that they are sufficient. In Section 5 we deal with the triangle-free 2 -factor problem, that is, with the case when $k=3$. We characterize the facet-inducing bipartition inqualities for this case. In fact, we prove the conjecture of the previous section for $k=3$. Our results also show that, for $k=3$, the characterization is a good one, as the conditions can be checked by solving a few bipartite matching problems. Finally, we show that the known classes of inequalities are still not sufficient to define the the triangle-free 2-factor polytope. 


\section{Preliminaries}

We shall use the word factor to mean $k$-restricted 2-factor, whenever it is possible to do so without confusion. It is convenient to treat the optimal factor problem as a problem on a simple complete graph. This has the slight disadvantage that we lose the distinction among the cases $k=0,1,2$, but our contributions do not apply to these cases anyway. So we work with the complete graph $K_{n}=(V, E)$, where $|V|=n \geq 4$, and we write elements of $E$ as $(i, j)$ or $i j$. Notice that $i j=j i$.

For $S \subseteq V$, we denote $V \backslash S$ by $\bar{S}$. For $S, T \subseteq V, E(S: T)$ denotes $\{i j \in E: i \in S, j \in$ $T\}$. For $S \subseteq V, E(S)$ denotes $E(S: S)$ and $\delta(S)$ denotes $E(S: \bar{S})$. For $v \in V$, we may abbreviate $\delta(\{v\})$ to $\delta(v)$. For $B \subseteq E$ and $x \in \mathbf{R}^{E}, x(B)$ denotes $\sum\left(x_{i j}: i j \in B\right)$. We may write $x(S)$ instead of $x(E(S))$ for $S \subseteq V$ whenever no confusion arises. Generally, we do not distinguish between a subset $C$ of $E$ and its incidence vector $x \in\{0,1\}^{E}$ defined by $x_{i j}=1$ if and only if $i j \in C$.

Given $c \in \mathbf{R}^{E}$, the following is an integer linear programming formulation of the optimal factor problem.

$$
\begin{array}{rc}
\operatorname{minimize} & \sum\left(c_{i j} x_{i j}: i j \in E\right) \\
\text { subject to } & x(\delta(v))=2, \quad v \in V ; \\
\text { (1a) } & x(S) \leq|S|-1, \quad S \subset V \text { and } 2 \leq|S| \leq k ; \\
\text { (1b) } & x_{i j} \geq 0, \quad i j \in E ; \\
\text { (1c) } & x_{i j} \text { integer, } \quad i j \in E .
\end{array}
$$

The constraints (1a) are called degree constraints and (1b) are called subtour elimination (SE) constraints. A factor is the union of node-disjoint cycles, called subtours, covering all nodes in $K_{n}$, and moreover, each subtour contains more than $k$ edges.

The convex hull of feasible solutions to (1) is a bounded polyhedron, which we denote by $P^{k}$. An inequality $f x \leq f_{0}$ is said to be valid for $P^{k}$ if $f x \leq f_{0}$ holds for all $x \in P^{k}$. The inequality $f x \leq f_{0}$ is face inducing if it is valid and there exists a point $x^{*} \in P^{k}$ such that $f x^{*}=f_{0}$. If, moreover, there exists an affinely independent set of $\operatorname{dim}\left(P^{k}\right)$ such points, then it is facet inducing. 


\section{Bipartition inequalities}

In this section, a large class of inequalities, called bipartition inequalities, will be shown to be valid for $P^{k}$. This class was first introduced by Boyd and Cunningham [1] for the TSP polytope as a generalization of the clique-tree class.

Let $\mathcal{H}=\left\{H_{1}, \ldots, H_{h}\right\}$ be a collection of mutually disjoint subsets of $V$ called handles, and let $T_{1}, T_{2}, \ldots, T_{t+m}$ be mutually disjoint proper subsets of $V$ called teeth. A tooth is called degenerate if it is contained in the union of the handles; otherwise it is called nondegenerate. Assume that no $T_{j}$ is contained in any $H_{i}$, and that $T_{j}$ is nondegenerate if and only if $1 \leq j \leq t$. Assume also that each handle $H_{i}$ intersects $2 k_{i}+1$ teeth, where $k_{i}$ is a positive integer, and $d_{j}$ denotes the number of handles intersected by tooth $T_{j}$ for all $j, 1 \leq j \leq t+m$. The bipartition inequality associated with these handles and teeth is given by

$$
\begin{aligned}
& \sum_{i=1}^{h} x\left(H_{i}\right)+\sum_{j=1}^{t} x\left(T_{j}\right)+\sum_{j=t+1}^{t+m} \frac{d_{j}}{d_{j}-1} x\left(T_{j}\right) \\
& \leq \sum_{i=1}^{h}\left|H_{i}\right|+\sum_{i=1}^{h} k_{i}+\sum_{j=1}^{t}\left(\left|T_{j}\right|-d_{j}-1\right)+\sum_{j=t+1}^{t+m} \frac{d_{j}}{d_{j}-1}\left(\left|T_{j}\right|-d_{j}\right) .
\end{aligned}
$$

Notice that in the special case when $h=0, t=1$, and $m=0$, the bipartition inequality is just an SE inequality. In the special case when $h=1$ and $T_{j} \cap H_{1} \neq \emptyset$ for all $j$, is the well-known comb inequality. Finally, when $m=0$ and the intersection graph of the $H_{i}$ and $T_{j}$ form a tree, we have the clique-tree inequalities of Grötschel and Pulleyblank [8]. Recently, Carr [2] has shown that, when the number of handles and teeth are fixed, there is a polynomial-time algorithm to solve the separation problem for bipartition inequalities.

Figure 1 represents two bipartition inequalities. The hollow nodes, labelled $v_{0}$ and $v_{0}^{\prime}$ in the handles, represent optional nodes that may or may not exist. In the case when the optional nodes do not exist, both inequalities have right-hand side $c_{0}=8$. The coefficient $c_{e}$ of the left-hand side depends on the total weight of the sets that contain $e$. Weights of 1 are omitted for simplicity, and thus only the weights of degenerate teeth are given. For instance, $c_{v v^{\prime}}=c_{s s^{\prime}}=2$ for the inequality on the left and $c_{v v^{\prime}}=2, c_{s s^{\prime}}=0$ and $c_{r w}=1$ for the one on the right. 

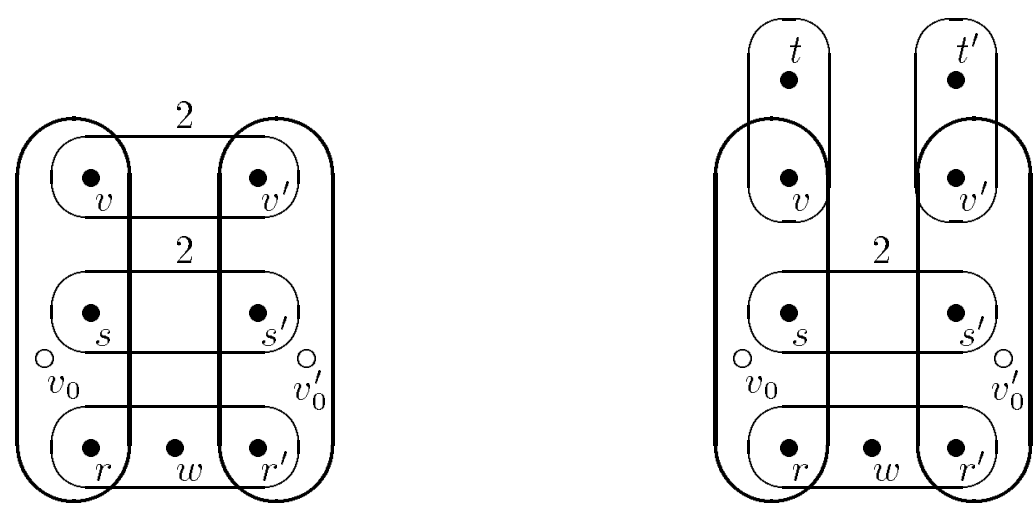

Figure 1: Two bipartition inequalities

A $k$-bipartition inequality is one for which every tooth has size at most $k$. The main result of this section is the following.

Theorem 3.1 Any k-bipartition inequality $c x \leq c_{0}$ is valid for $P^{k}$.

For the remainder of this section $c x \leq c_{0}$ denotes a $k$-bipartition inequality defined by (2). Consider the following maximization problem:

$$
z^{*}(c)=\max \left\{c x: x \in P^{k}\right\} .
$$

A factor $x^{*}$ is said to be c-optimal if $c x^{*}=z^{*}(c)$, and is $c$-tight if $c x^{*}=c_{0}$. The inequality $c x \leq c_{0}$ is valid for $P^{k}$ if and only if $z^{*}(c) \leq c_{0}$ and is face-inducing if and only $z^{*}(c)=c_{0}$. We will show the validity of $c x \leq c_{0}$ by induction on the number of handles defining $c x \leq c_{0}$. To do so, we first apply a procedure to transform a $c$-optimal factor $x^{*}$ to into a $c$-optimal factor $\hat{x}$ having a special structure. Then, we "decompose" $c x \leq c_{0}$ with respect to $\hat{x}$ into two bipartition inequalities with smaller number of defining handles, and use induction.

For any factor $x$, let $\kappa(x)$ denote the number of subtours in $x$. Given any subset $S$ of $V$ and a factor $x$ such that $x(E(S))<|S|-1$, we can apply the following procedure $2 O P T$. This will be used repeatedly in our polyhedral proofs. It is analogous to the well-known local optimization procedure of the same name. In fact, we will need it only 
for the case where $S=T_{j}$ or $S=H_{i} \cap T_{j}$, where $T_{j}$ is a tooth and $H_{i}$ is a handle of the bipartition inequality $c x \leq c_{0}$.

\section{Procedure 2OPT}

Given: a vector $c \in \mathbf{R}^{E}$, a factor $x$, and a subset $S$ of $V$ such that $x(E(S))<|S|-1$.

Step 0. If there is exactly one subtour of $x$ that visits $S$, then go to Step 1; else go to Step 2.

Step 1. A subtour of $x$ contains a path $u v \cdots p q \cdots u^{\prime} v^{\prime}$ such that $v, p, v^{\prime} \in S$ and $u, q, u^{\prime} \in \bar{S}$. Replace $u v, u^{\prime} v^{\prime}$ in $x$ by $u u^{\prime}$ and $v v^{\prime}$ to obtain $\bar{x}$. STOP.

Step 2. Choose a pair of edges $u v, u^{\prime} v^{\prime}$ that are in different subtours of $x$, with $v, v^{\prime} \in S$ and $u, u^{\prime} \in V \backslash S$, such that $c_{u v}+c_{u^{\prime} v^{\prime}}$ is minimum over all such pairs. Replace $u v$ and $u^{\prime} v^{\prime}$ by $u u^{\prime}$ and $v v^{\prime}$ to obtain $\bar{x}$. STOP.

It is easy to see that 2OPT has the following property.

Proposition 3.2 2OPT terminates with a factor $\bar{x}$ such that $\bar{x}(E(S))>x(E(S))$ and $\kappa(\bar{x}) \leq \kappa(x)$. Moreover, $c \bar{x} \geq c x$ if and only if $c_{u v}+c_{u^{\prime} v^{\prime}} \leq c_{u u^{\prime}}+c_{v v^{\prime}}$.

Lemma 3.3 If $T$ is a tooth and $H$ is a handle of a bipartition inequality $c x \leq c_{0}$ and 2OPT is applied with $x$ a c-optimal factor $x^{*}$ and $S=T \cap H$, then 2OPT replaces an edge in $E(T \cap H: T \backslash H)$ and an edge in $E(T \cap H: H \backslash T)$ by an edge in $E(T \cap H)$ and an edge in $E(T \backslash H: H \backslash T)$. The resulting factor $\hat{x}$ is also c-optimal.

Proof: 2OPT replaces a pair of edges $u v$ and $u^{\prime} v^{\prime}$ in $\delta(S)$ with $u u^{\prime}$ and $v v^{\prime}$, where $v v^{\prime} \in E(S)$. By definition of $c, c_{u v}+c_{u^{\prime} v^{\prime}} \leq c_{v v^{\prime}}$. Since $x^{*}$ is c-optimal, this inequality must hold with equality, and moreover $c_{u u^{\prime}}=0$. It follows that $u u^{\prime} \in E(H \backslash S: T \backslash S)$, proving the lemma.

Lemma 3.4 Let $x^{*}$ be a c-optimal factor. Then there exists a c-optimal factor $\hat{x}$ such that

(a) $\hat{x}\left(H_{i} \cap T_{j}\right)=\left|H_{i} \cap T_{j}\right|-1$ for all $i$ and $j$ with $H_{i} \cap T_{j} \neq \emptyset$;

(b) $\hat{x}\left(H_{i}\right)=x^{*}\left(H_{i}\right)$ and $\hat{x}\left(T_{j}\right)=x^{*}\left(T_{j}\right)$ for all $i$ and $j$; 
(c) $x^{*}\left(\delta\left(H_{i}\right) \cap\left\{e: c_{e}=0\right\}\right) \leq \hat{x}\left(\delta\left(H_{i}\right) \cap\left\{e: c_{e}=0\right\}\right)$ for all $H_{i} \in \mathcal{H}$.

Proof: Apply 2OPT with $S=H_{i} \cap T_{j}$ for any handle-tooth pair violating (a). It follows from Lemma 3.3 that (b) and (c) are satisfied by the resulting factor $\hat{x}$. We can repeat this until (a) is also satisfied.

For any $S \subseteq V$, a factor $x$ is said to saturate $S$ if $x(S)=|S|-1$. We say that a factor $\hat{x}$ is simple if it satisfies condition (a) of Lemma 3.4 .

We describe a decomposition of $c x \leq c_{0}$ relative to a fixed handle $H$ and a fixed $c$-optimal simple factor $\hat{x}$. This construction will be used not only in the proof of Theorem 3.1, but in the proofs of subsequent results. Let $D$ be the index set of the degenerate teeth, and let $N$ be the index set of the nondegenerate teeth. For a fixed $H \in \mathcal{H}$, define $S_{j} \equiv T_{j} \cap H$ for all $j$ with $T_{j} \cap H \neq \emptyset$. Let $J^{\prime}$ denote the index set of the teeth that intersect $H$, and define $J$ to be $\left\{j \in J^{\prime}: d_{j} \geq 3\right.$, or $d_{j}=2$ and $\left.j \in N\right\}$. With respect to the factor $\hat{x}$ and $H$, define $J^{0}(\hat{x})$ to be $\left\{j \in J: \hat{x}\left(S_{j}: T_{j} \backslash S_{j}\right)=0\right\}$, and $J^{+}(\hat{x})$ to be $J \backslash J^{0}(\hat{x})$. Define the following set of teeth relative to $\hat{x}$ and $H$ : for every $j \in J^{0}(\hat{x})$, $T_{j}^{\prime}=S_{j} \cup\left\{v_{j}\right\}$, where $v_{j}$ is a fixed element of $T_{j} \backslash S_{j}$; for every $j \in J^{+}(\hat{x}), T_{j}^{\prime}=S_{j} \cup\left\{v_{j}\right\}$, where $v_{j}$ is the element of $T_{j} \backslash S_{j}$ satisfying $\hat{x}_{v_{j} s}=1$ for some $s \in S_{j}$, and $T_{j}^{\prime}=T_{j}$ for all $j \in J^{\prime} \backslash J$. By definition and the property of $\hat{x}$, we have

(4) $\quad \hat{x}\left(T_{j}^{\prime}\right)=\hat{x}\left(S_{j}\right)=\left|S_{j}\right|-1=\left|T_{j}^{\prime}\right|-2$, for all $j \in J^{0}(\hat{x})$;

(5) $\quad \hat{x}\left(T_{j}^{\prime}\right)=\left|T_{j}^{\prime}\right|-1=\left|S_{j}\right|$, for all $j \in J^{+}(\hat{x})$.

We now construct two bipartition inequalities from $c x \leq c_{0}$. Let $a x \leq a_{0}$ be the bipartition inequality obtained from $c x \leq c_{0}$ by deleting the handle $H$ and all the teeth that intersect only $H$, and replacing $T_{j}$ by $T_{j} \backslash S_{j}$ for each $j \in J^{0}(\hat{x})$. Let $b x \leq b_{0}$ be a comb inequality defined by the handle $H$ and all teeth $T_{j}^{\prime}$. So $a x \leq a_{0}$ and $b x \leq b_{0}$ are $k$-bipartition inequalities with fewer than $h$ handles.

Using (4) and (5), we can now express $c \hat{x}$ and $c_{0}$ in terms of $a \hat{x}+b \hat{x}$ and $a_{0}+b_{0}$, respectively, as follows

$$
\begin{aligned}
c \hat{x} & =a \hat{x}+b \hat{x}+\gamma_{1}+\gamma_{2}+\gamma_{3}, \quad \text { where } \\
\gamma_{1} & \equiv \sum\left(-\frac{\hat{x}\left(T_{j} \backslash S_{j}\right)}{\left(d_{j}-1\right)\left(d_{j}-2\right)}+\frac{\hat{x}\left(T_{j}^{\prime}\right)}{\left(d_{j}-1\right)}: j \in J^{0}(\hat{x}) \cap D\right),
\end{aligned}
$$




$$
\begin{aligned}
\gamma_{2} & \equiv \sum\left(\frac{\hat{x}\left(T_{j}\right)}{d_{j}-1}-\hat{x}\left(T_{j}^{\prime}\right): j \in J^{+}(\hat{x}) \cap D\right), \\
\gamma_{3} & \equiv \sum\left(-\hat{x}\left(T_{j}^{\prime}\right): j \in J^{+}(\hat{x}) \cap N\right) . \\
c_{0} & =a_{0}+b_{0}+\gamma_{1}^{\prime}+\gamma_{2}^{\prime}+\gamma_{3}^{\prime}, \quad \text { where } \\
\gamma_{1}^{\prime} & \equiv \sum\left(\frac{d_{j}}{d_{j}-1}\left(\left|T_{j}\right|-d_{j}\right)-\frac{d_{j}-1}{d_{j}-2}\left(\left|T_{j} \backslash S_{j}\right|-d_{j}+1\right)-\left|S_{j}\right|+1: j \in J^{0}(\hat{x}) \cap D\right) \\
\gamma_{2}^{\prime} & \equiv \sum\left(\frac{d_{j}}{d_{j}-1}\left(\left|T_{j}\right|-d_{j}\right)-\left|T_{j}\right|+d_{j}-\left|S_{j}\right|+1: j \in J^{+}(\hat{x}) \cap D\right), \\
\gamma_{3}^{\prime} & \equiv \sum\left(-\left|S_{j}\right|: j \in J^{+}(\hat{x}) \cap N\right)
\end{aligned}
$$

Lemma 3.5 Let $\hat{x}$ be a simple c-optimal factor. Suppose that $a \hat{x} \leq a_{0}$ and $b \hat{x} \leq b_{0}$ hold. Then $c \hat{x} \leq c_{0}$ also holds, and moreover, $c \hat{x}=c_{0}$ if and only if $a \hat{x}=a_{0}, b \hat{x}=b_{0}$ and $\gamma_{i}=\gamma_{i}^{\prime}$ for $i=1,2,3$.

Proof: It suffices to show that $\gamma_{i} \leq \gamma_{i}^{\prime}, i=1,2,3$. Note first that by $(5), \gamma_{3}=\gamma_{3}^{\prime}$. Next, observe that for $j \in D \cap J^{0}(\hat{x})$, we have

$$
\hat{x}\left(T_{j} \backslash S_{j}\right) \geq \sum\left(\left|T_{j} \cap H_{i}\right|-1: H_{i} \in \mathcal{H} \backslash\{H\}, H_{i} \cap T_{j} \neq \emptyset\right)=\left|T_{j} \backslash S_{j}\right|-d_{j}+1 .
$$

It follows from (4) that

$$
\gamma_{1} \leq \sum\left(-\frac{\left|T_{j} \backslash S_{j}\right|-d_{j}+1}{\left(d_{j}-1\right)\left(d_{j}-2\right)}+\frac{\left|S_{j}\right|-1}{\left(d_{j}-1\right)}: j \in J^{0}(\hat{x}) \cap D\right)=\gamma_{1}^{\prime},
$$

and from (5) that

$$
\gamma_{2} \leq \sum\left(\frac{\left|T_{j}\right|-1}{d_{j}-1}-\left|S_{j}\right|: j \in J^{+}(\hat{x}) \cap D\right)=\gamma_{2}^{\prime} .
$$

Lemma 3.5 seems to say that there are other inequalities that hold with equality whenever $c x \leq c_{0}$ does. However, what it really says is that for each factor satisfying $c x \leq c_{0}$ with equality, one can define other inequalities that the same factor also satisfies with equality. The new inequalities depend on the given point. We are now ready to prove the validity of the bipartition inequalities. 
Proof of Theorem 3.1. We prove the theorem by induction on the number $h$ of handles defining $c x \leq c_{0}$. For $h=0$, the inequality is the sum of SE constraints $x\left(\delta\left(T_{j}\right)\right) \leq\left|T_{j}\right|-1$, where $\left|T_{j}\right| \leq k$. For $h=1$, the inequality $c x \leq c_{0}$ is a comb with a handle $H$ intersected by all teeth $T_{1}, \ldots, T_{2 k_{h}+1}$ of size at most $k$. To prove its validity, we use the usual technique known for the TSP. We add the inequalities:

$$
\begin{aligned}
& \frac{1}{2} x(\delta(v))=1, \quad \text { for all } v \in H, \\
& \frac{1}{2} x\left(T_{j}\right) \leq \frac{1}{2}\left(\left|T_{j}\right|-1\right), \quad j=1, \ldots, 2 k_{h}+1 \\
& \frac{1}{2} x\left(T_{j} \backslash H\right) \leq \frac{1}{2}\left(\left|T_{j} \backslash H\right|-1\right), \quad \text { for all } j \text { such that }\left|T_{j} \backslash H\right| \geq 2 \\
& \frac{1}{2} x\left(T_{j} \cap H\right) \leq \frac{1}{2}\left(\left|T_{j} \cap H\right|-1\right), \quad \text { for all } j \text { such that }\left|T_{j} \cap H\right| \geq 2
\end{aligned}
$$

Taking the integer part of each coefficient and the right-hand side of the resulting inequality yields $c x \leq c_{0}$.

Assume now that the theorem holds for the number of handles less than $h$. By Lemma 3.4 , it suffices to check the validity of $c x \leq c_{0}$ for any simple $c$-optimal solution $\hat{x}$ to $(3)$. With respect to $\hat{x}$, we can construct as above $a x \leq a_{0}, b x \leq b_{0}$, as well as $\gamma_{i}, \gamma_{i}^{\prime}, i=1,2,3$. By the induction hypothesis, $a x \leq a_{0}$ and $b x \leq b_{0}$ are valid for $P^{k}$, and therefore $c x \leq c_{0}$ is valid by Lemma 3.5.

Similar methods allow us to establish some further properties of $c$-tight factors. These properties will be useful later. They are new even for the TSP. The first one indicates that there are exactly two ways for a tight factor to traverse a degenerate tooth. These are indicated in Figure 2.

Theorem 3.6 Let $T$ be a degenerate tooth, let $H_{1}, H_{2}, \ldots, H_{d}$ be the handles intersecting $T$, and let $x^{*}$ be a c-tight factor. If $x^{*}$ does not saturate $T$, then $x^{*}(T)=|T|-d$ and $x^{*}\left(T \cap H_{i}\right)=\left|T \cap H_{i}\right|-1$ for $i=1, \ldots, d$.

Proof: First, we show that $x^{*}(T)=|T|-d$. Let $\hat{x}$ be the simple factor produced from $x^{*}$ via Lemma 3.4 . It will be enough to prove that $\hat{x}(T)=|T|-d$. We may assume that 

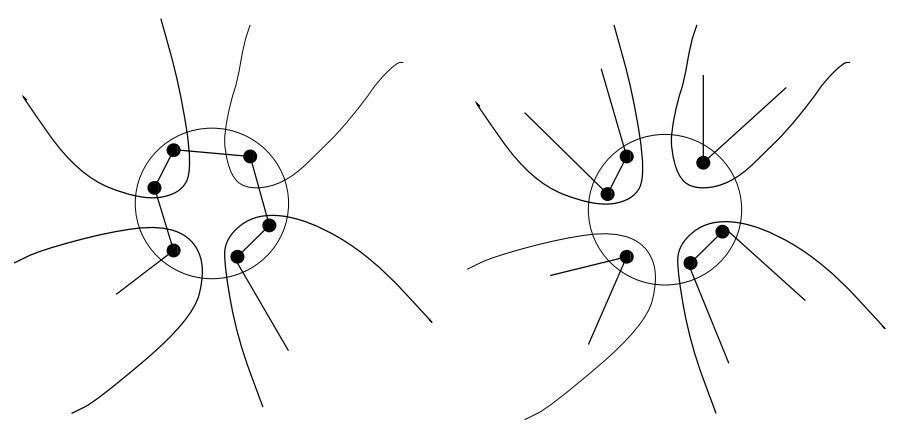

Figure 2: The two ways of traversing a degenerate tooth

$d \neq 2$, since if $d=2$, then $\hat{x}(T)=|T|-2$, and we are done. Since $\hat{x}$ does not saturate $T$, there is a proper nonempty subset $A$ of $T$ such that $\hat{x}(A: T \backslash A)=0$. Suppose first that both $A$ and $T \backslash A$ intersect at least two handles. Form the bipartition inequality $c^{\prime} x \leq c_{0}^{\prime}$ by replacing the tooth $T$ by the teeth $A, T \backslash A$. It is easy to see that $c_{0}^{\prime}=c_{0}$, so $c^{\prime} \hat{x} \leq c_{0}$, by Theorem 3.1. But it is also easy to see that $c^{\prime} \hat{x}>c \hat{x}$, since the coefficient of any edge $e \in E(T)$ for which $\hat{x}_{e}>0$ has increased. This contradicts the $c$-tightness of $\hat{x}$. Therefore, we may assume that one of $A$ or $T \backslash A$, say $A$, is of the form $H \cap T$ for some handle $H$. For this $H$ we can apply the decomposition procedure to $c x \leq c_{0}$, and we will have $T=T_{j}$, where $j \in J^{0}(\hat{x})$. By Lemma 3.5, we have $\gamma_{1}=\gamma_{1}^{\prime}$, and thus by (4), (7), $\hat{x}$ satisfies (6) with equality. Finally, using Lemma 3.4, we have

$$
x^{*}(T)=\hat{x}(T)=\hat{x}(A)+\hat{x}(T \backslash A)=(|A|-1)+|T \backslash A|-d+1=|T|-d,
$$

as required.

Now suppose that $x^{*}\left(T \cap H_{i}\right)<\left|T \cap H_{i}\right|-1$ for some $H_{i}$ intersecting $T$. Apply 2OPT relative to $x^{*}$ and $S=T \cap H_{i}$. Note that by Lemma 3.3, the resulting $\bar{x}$ must contain an edge $e_{0} \in \delta(T)$ with $c_{e_{0}}=0$, and moreover satisfy $c \bar{x}=c x^{*}$ and $\bar{x}(T)=x^{*}(T)<|T|-1$. So we can apply 2OPT again relative to $\bar{x}$ and $S=T$. Then 2 OPT replaces $e_{0}, e_{1} \in \delta(T)$ with $e \in E(T)$ and $e^{\prime} \in E(V \backslash T)$. Since

$$
c_{e_{0}}+c_{e_{1}} \leq 1<\frac{d_{j}}{d_{j}-1} \leq c_{e}+c_{e^{\prime}},
$$

the new factor $\tilde{x} \in P^{k}$ satisfies $c \tilde{x}>c \bar{x}=c_{0}$, a contradiction.

The second result states that there are just two ways in which a tight factor can traverse a handle, which we indicate in Figure 3. 

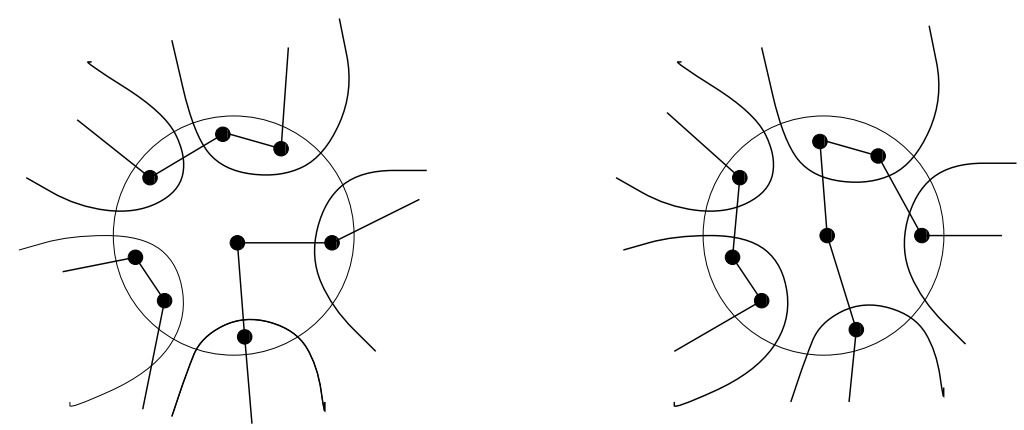

Figure 3: The two ways of traversing a handle

Theorem 3.7 Let $x^{*}$ be a c-tight factor, let $H$ be a handle, and let $2 k_{h}+1$ be the number of teeth intersecting $H$. Then exactly one of the following is true:

(a) $x^{*}(H)=|H|-k_{h}-1, x^{*}(\delta(H))=2 k_{h}+2$, and $\left|\left\{e \in \delta(H): x_{e}^{*}>0<c_{e}\right\}\right| \geq 2 k_{h}+1$;

(b) $x^{*}(H)=|H|-k_{h}, x^{*}(\delta(H))=2 k_{h}$, and $\left|\left\{e \in \delta(H): x_{e}^{*}>0<c_{e}\right\}\right|=2 k_{h}$.

Proof: It is easy to see that, if the simple $c$-tight factor $\hat{x}$ obtained from $x^{*}$ via Lemma 3.4 satisfies the conclusions of the theorem, then so too does $x^{*}$. Therefore, we deal with $\hat{x}$. If we decompose $c x \leq c_{0}$ relative to $\hat{x}$ and the handle $H$, we get the comb inequality $b x \leq b_{0}$ defined by

$$
x(H)+\sum_{j=1}^{2 k_{h}+1} x\left(T_{j}^{\prime}\right) \leq|H|+\sum_{j=1}^{2 k_{h}+1}\left(\left|T_{j}^{\prime}\right|-1\right)-k_{h}-1 .
$$

Let $s^{*}$ be the number of teeth $T_{j}^{\prime}$ satisfying $\hat{x}\left(T_{j}^{\prime}\right)=\left|T_{j}^{\prime}\right|-1$. Then $s^{*} \leq \hat{x}(\delta(H))$, and also from its definition, $s^{*} \leq 2 k_{h}+1$. By Lemma $3.5, b \hat{x}=b_{0}$, that is, (9) holds with equality for $x=\hat{x}$, so

$$
\begin{aligned}
\hat{x}(H) & =|H|-k_{h}-1+\sum_{j=1}^{2 k_{h}+1}\left(\left|T_{j}^{\prime}\right|-1-\hat{x}\left(T_{j}^{\prime}\right)\right) \\
& \geq|H|-k_{h}-1+\left(2 k_{h}+1-s^{*}\right)=|H|+k_{h}-s^{*}
\end{aligned}
$$

Therefore, $2 s^{*} \geq 2 k_{h}-2 \hat{x}(H)+2|H|=2 k_{h}+\hat{x}(\delta(H)) \geq 2 k_{h}+s^{*}$. It follows that $s^{*} \geq 2 k_{h}$, and therefore that $s^{*}=2 k_{h}$ or $2 k_{h}+1$. If $s^{*}=2 k_{h}$, then from (10), we get that $\hat{x}(H)=|H|-k_{h}$, and then from the identity $\hat{x}(H)+\hat{x}(\delta(H))=2|H|$, that $\hat{x}(\delta(H))=2 k_{h}$, so (b) holds. If $s^{*}=2 k_{h}+1$, then we can show in the same way that (a) holds. 


\section{Degenerate cuts}

In this section we describe an important necessary condition for a bipartition inequality to be facet-inducing. We begin with some simple examples.

Suppose that the intersection graph of the $H_{i}, T_{j}$ is not connected, for example, that there is no handle and more than one tooth. Then it is easy to see that the inequality is the sum of the inequalities corresponding to the connected components, and thus is contained in the faces induced by those inequalities. There is one very special case in which such a bipartition inequality can be facet-inducing, namely, if there are just two teeth which are complements of each other. Then the inequality induces the same face as does the SE inequality determined by one of the teeth.

As a second example, suppose there are just two handles and every tooth intersecting both of them is degenerate. Then it is easy to see that the inequality is the sum of two comb inequalities, each defined by one of the handles and the teeth that intersect it. Again, there is one case in which such an inequality can be facet-inducing, namely, when the two handles are complements of each other, for then the inequality induces the same face as each of the comb inequalities.

The above examples both have the property that there is a set $W \subset V$ such that each of $W, \bar{W}$ contains at least one node in some tooth or handle, and no handle or nondegenerate tooth intersects both $W$ and $\bar{W}$. In this situation we call the set of degenerate teeth intersecting both $W$ and $\bar{W}$ a degenerate cut. The main result of this section is the following.

Theorem 4.1 Let $c x \leq c_{0}$ be a bipartition inequality that does not have two complementary handles or teeth. If $c x \leq c_{0}$ has a degenerate cut, then it does not define a facet of $P^{k}$

Notice that it follows from the theorem that a bipartition inequality having a tree as its intersection graph and having a degenerate tooth (and not having two complementary handles), cannot be facet-inducing. It is possible to prove this fact by showing that such an inequality is a non-negative linear combination of $d$ other bipartition inequalities, where $d$ is the number of handles intersected by the degenerate tooth. For more general bipartition inequalities, it need not be true that if there is a degenerate cut, then the 
inequality is a non-negative combination of other bipartition inequalities. This may suggest why the proof of Theorem 4.1 is more difficult than one might expect.

For the remainder of this section, $c x \leq c_{0}$ denotes a bipartition inequality containing a degenerate cut determined by $W \subset V$. Let $C$ be the index set of the teeth intersecting both $W$ and $\bar{W}$. For each $j \in C$, let $d_{j}^{\prime}$ denote the number of handles that are intersected by $T_{j}$ and contained in $W$, and let $d_{j}^{\prime \prime}$ denote the number of handles that are intersected by $T_{j}$ and contained in $\bar{W}$. Clearly, $d_{j}=d_{j}^{\prime}+d_{j}^{\prime \prime}$. Define $a x \leq a_{0}$ to be the bipartition inequality whose handles are the handles contained in $W$ and whose teeth are the teeth not contained in $\bar{W}$, and $b x \leq b_{0}$ to be the bipartition inequality whose handles are the handles contained in $\bar{W}$ and whose teeth are the teeth not contained in $W$. It is easy to verify that $c x \leq c_{0}$ can be represented as:

$$
a x+b x-\sum\left(\frac{d_{j}-2}{d_{j}-1} x\left(T_{j}\right): j \in C\right) \leq a_{0}+b_{0}-\sum\left(\frac{d_{j}-2}{d_{j}-1}\left(\left|T_{j}\right|-1\right): j \in C\right) .
$$

Lemma 4.2 Any c-tight solution $x^{*}$ satisfies

$$
\begin{aligned}
& a x^{*}+2 b x^{*}-\sum\left(\left[\frac{d_{j}^{\prime \prime}-1}{d_{j}-1}+2 \frac{d_{j}^{\prime}-1}{d_{j}-1}\right] x^{*}\left(T_{j}\right): j \in C\right) \\
= & a_{0}+2 b_{0}-\sum\left(\left[\frac{d_{j}^{\prime \prime}-1}{d_{j}-1}+2 \frac{d_{j}^{\prime}-1}{d_{j}-1}\right]\left(\left|T_{j}\right|-1\right): j \in C\right) .
\end{aligned}
$$

Proof: Let $I\left(x^{*}\right)$ denote $\left\{j \in C: x^{*}\left(T_{j}\right)=\left|T_{j}\right|-d_{j}\right\}$. By Lemma 3.6, we have $x^{*}\left(T_{j}\right)=\left|T_{j}\right|-1$ for all $j \in C \backslash I\left(x^{*}\right)$, and $x\left(T_{j} \cap H_{i}\right)=\left|T_{j} \cap H_{i}\right|-1$ for all $j \in I\left(x^{*}\right)$ and $i$ such that $H_{i}$ intersects $T_{j}$.

For every $j \in I\left(x^{*}\right)$, choose a handle $H_{i} \subset \bar{W}$ such that $H_{i}$ intersects $T_{j}$ and define $T_{j}^{\prime}$ to be $\left(T_{j} \cap W\right) \cup H_{i}$. Define $\hat{a} x \leq \hat{a}_{0}$ to be the bipartition inequality obtained from ax $\leq a_{0}$ by replacing $T_{j}$ by $T_{j}^{\prime}$ for each $j \in I\left(x^{*}\right)$. Notice that $x^{*}\left(T_{j}^{\prime}\right)=\left|T_{j}^{\prime}\right|-d_{j}^{\prime}-1$ for all $j \in I\left(x^{*}\right)$. Also notice that each $T_{j}^{\prime}$ is a nondegenerate tooth of $\hat{a} x \leq \hat{a}_{0}$. Therefore, $\hat{a} x$ is just $a x+\sum\left(x\left(T_{j}^{\prime}\right)-x\left(T_{j}\right): j \in I\left(x^{*}\right)\right)$ and $\hat{a}_{0}$ is just $a_{0}+\sum\left(\left|T_{j}^{\prime}\right|-\left|T_{j}\right|: j \in I\left(x^{*}\right)\right)$. Since $\hat{a} x^{*} \leq \hat{a}_{0}$, we have

$$
\begin{aligned}
a x^{*} & \leq \hat{a}_{0}-\sum\left(x^{*}\left(T_{j}^{\prime}\right)-x^{*}\left(T_{j}\right): j \in I\left(x^{*}\right)\right) \\
& =a_{0}+\sum\left(\left|T_{j}^{\prime}\right|-\left|T_{j}\right|: j \in I\left(x^{*}\right)\right)-\sum\left(\left|T_{j}^{\prime}\right|-d_{j}^{\prime}-1-\left(\left|T_{j}\right|-d_{j}\right): j \in I\left(x^{*}\right)\right) \\
& =a_{0}-\sum\left(d_{j}-d_{j}^{\prime}-1: j \in I\left(x^{*}\right)\right) \\
& =a_{0}-\sum\left(d_{j}^{\prime \prime}-1: j \in I\left(x^{*}\right)\right) .
\end{aligned}
$$


Therefore

$$
a x^{*} \leq a_{0}-\sum\left(d_{j}^{\prime \prime}-1: j \in I\left(x^{*}\right)\right)
$$

By symmetry we have

$$
b x^{*} \leq b_{0}-\sum\left(d_{j}^{\prime}-1: j \in I\left(x^{*}\right)\right) .
$$

Adding (13) and (14), we get

$$
a x^{*}+b x^{*} \leq a_{0}+b_{0}-\sum\left(d_{j}-2: j \in I\left(x^{*}\right)\right) .
$$

Since $c x^{*}=c_{0}$, we have from (11) that

$$
a x^{*}+b x^{*}-\sum\left(\frac{d_{j}-2}{d_{j}-1} x^{*}\left(T_{j}\right): j \in C\right)=a_{0}+b_{0}-\sum\left(\frac{d_{j}-2}{d_{j}-1}\left(\left|T_{j}\right|-1\right): j \in C\right) .
$$

Since $x^{*}\left(T_{j}\right)=\left|T_{j}\right|-1$ for all $j \in C \backslash I\left(x^{*}\right)$, it follows that (15) holds with equality, and therefore that (13) and (14) also hold with equality. Now from this and the facts that $x^{*}\left(T_{j}\right)=\left|T_{j}\right|-1$ for $j \in C \backslash I\left(x^{*}\right)$ and $x^{*}\left(T_{j}\right)=\left|T_{j}\right|-d_{j}$ for $j \in I\left(x^{*}\right)$, the truth of (12) follows by a straightforward calculation.

We have shown that each point $x^{*}$ of $P^{k}$ such that $c x^{*}=c_{0}$ satisfies an additional equation (12), which we denote as $g x=g_{0}$. We can show that the set $F$ of such points is not a facet, by showing that $g x=g_{0}$ is not a linear combination of $c x=c_{0}$ and equations that are satisfied by all points of $P^{k}$. The latter equations are described as follows.

Lemma 4.3 The degree constraints (1a) constitute a minimal equality system for $P^{k}$.

Proof: It is well known (and easy to prove) that the degree constraints define a minimal equality system for the TSP polytope. Since $P^{k}$ contains the TSP polytope and the degree constraints are valid for $P^{k}$, the result follows.

Proof of Theorem 4.1: Let $A$ be the node-edge incidence matrix of $K_{n}$, so $A x=\mathbf{2}$ is the system of degree constraints. We must show that $g$, or equivalently $g^{\prime}=c-g$, is not in the row space of $\left(\begin{array}{l}A \\ c\end{array}\right)$. Notice that

$$
g^{\prime} x=(c-g) x=b x-\sum_{j \in C} \frac{d_{j}^{\prime}-1}{d_{j}-1} x\left(T_{j}\right)
$$


Let $H_{1}$ be any handle in $W$ with intersecting teeth, say $T_{1}, T_{2}$ and $T_{3}$, and choose nodes $v \in H_{1} \cap T_{1}, u \in H_{1} \cap T_{2}, w \in H_{1} \cap T_{3}$. Let $c^{\prime} x \leq c_{0}^{\prime}$ be obtained from $c x \leq c_{0}$ by complementing $H_{1}$, that is,

$$
c^{\prime}=c-\frac{1}{2} \sum\left(A_{i}: i \in H_{1}\right)+\frac{1}{2} \sum\left(A_{i}: i \in V \backslash H_{1}\right), \quad c_{0}^{\prime}=c_{0}+\left|V \backslash H_{1}\right|-\left|H_{1}\right|,
$$

where $A_{i}$ is the row of $A$ indexed by node $i$. So we only need to show that $g^{\prime}$ is not in the row space of $\left(\begin{array}{l}A \\ c^{\prime}\end{array}\right)$.

Consider $B=\left\{v v^{\prime}: v^{\prime} \in V \backslash T_{1}\right\} \cup\left\{u w, u u^{\prime}: u^{\prime} \in T_{1} \backslash\{v\}\right\}$. Notice that $B$ consists of a spanning tree and an additional edge forming a triangle with $|B|=|V|$, and so the columns of $A$ indexed by $B$ are linearly independent, that is, $B$ forms a basis of $E$. Further observe that $g_{e}^{\prime}=c_{e}^{\prime}=0$ for all $e \in B$, and so what remains to show is that $c^{\prime}$ and $g^{\prime}$ are linearly independent. Since $c x \leq c_{0}$ does not have two complementary handles, by symmetry we may assume without loss of generality that $W$ itself is not a handle. Let $w^{\prime} \in W \backslash H_{1}$ and choose $\bar{w} \in \bar{W}$ with $c_{w^{\prime} \bar{w}}=0$. The proof is complete since $c_{w^{\prime} \bar{w}}^{\prime} \neq 0$ and $g_{w^{\prime} \bar{w}}^{\prime}=0$.

Now we describe a second necessary condition for a bipartition inequality to be facetinducing. It is simpler than the degenerate cut condition. However, it is not clear that it can be checked efficiently. The valid inequality $a x \leq a_{0}$ is dominated by the valid inequality $b x \leq b_{0}$ if every $x \in P^{k}$ satisfying $a x=a_{0}$ also satisfies $b x=b_{0}$. (Equivalently, the face induced by $a x \leq a_{0}$ is contained in the face induced by $b x \leq b_{0}$.)

Lemma 4.4 Let $c x \leq c_{0}$ be a $k$-bipartition inequality. If $c x \leq c_{0}$ is dominated by the nonnegativity constraint $-x_{e} \leq 0$ for some $e \in E$, then it is not facet inducing for $P^{k}$.

Proof. No subtour elimination constraint is dominated by $-x_{e} \leq 0$. So $c x \leq c_{0}$ is defined by at least one handle. If $c x \leq c_{0}$ has at least two handles, we choose a handle, say $H_{1}$ such that $e \notin E\left(H_{1}\right)$. If $c x \leq c_{0}$ is a comb inequality, then we can assume that it is defined to have handle $H_{1}$ with $e \notin E\left(H_{1}\right)$. Let $T_{1}, T_{2}$ and $T_{3}$ be the teeth intersecting $H_{1}$. Since $e \notin E\left(H_{1}\right)$, there exist two teeth, say $T_{1}$ and $T_{2}$, such that $e \notin \delta\left(T_{1} \cap H_{1}\right) \cup \delta\left(T_{2} \cap H_{1}\right)$. Let $c^{\prime} x \leq c_{0}^{\prime}$ be obtained from $c x \leq c_{0}$ by complementing $H_{1}$, see (16). Let $u \in T_{1} \cap H_{1}, v \in T_{2} \cap H_{1}, w \in T_{3} \cap H_{1}$. So $B=\left\{v v^{\prime}: v^{\prime} \in V \backslash T_{1}\right\} \cup\left\{u w, u u^{\prime}\right.$ : $\left.u^{\prime} \in T_{1} \backslash\{v\}\right\}$ forms a basis with $c_{h}^{\prime}=0$ for all $h \in B$. Since $e \notin B$ by construction of 
$B$ and $c^{\prime} x \leq c_{0}^{\prime}$ is not a multiple of $-x_{e} \leq 0, c x \leq c_{0}$ is not equivalent to $-x_{e} \leq 0$. It follows that $c x \leq c_{0}$ is not facet inducing.

We conjecture that the two necessary conditions are together sufficient for a bipartition inequality to be facet inducing.

Conjecture 4.5 Let $c x \leq c_{0}$ be a k-bipartition inequality having no complementary handle or tooth. Then $c x \leq c_{0}$ is facet-inducing for $P^{k}$ if and only if it has no degenerate cut and it is not dominated by a non-negativity inequality.

In the next section we prove this conjecture for $k=3$. In the process, we show that the second necessary condition can be checked efficiently.

\section{Facet-inducing bipartition inequalities for $k=3$}

In this section we characterize the 3 -bipartition inequalities that induce facets of $P^{3}$. In fact, we prove Conjecture 4.5 for the case when $k=3$.

Theorem 5.1 Let $c x \leq c_{0}$ be a 3-bipartition inequality having no complementary handle or tooth. Then $c x \leq c_{0}$ is facet-inducing for $P^{3}$ if and only if it has no degenerate cut and it is not dominated by a non-negativity inequality.

It is not at all obvious that the above characterization is a good one, in that it is not clear how easy it is to see that a given 3-bipartition inequality is not dominated by a nonnegativity inequality. We are going to show that this property is equivalent to a matching condition in a certain bipartite graph. This is Theorem 5.3 below. Theorem 5.3 not only shows that Theorem 5.1 is a good characterization; it also is essential in its proof.

For the remainder of this section $c x \leq c_{0}$ denotes a 3 -bipartition inequality. The condition that $c x \leq c_{0}$ is not dominated by a non-negativity inequality is equivalent to the condition that, for every edge $e$, there is a $c$-tight factor using $e$. As a preliminary to finding a condition for this, let us consider the problem of determining whether there is a $c$-tight factor at all. (In other words, is the inequality supporting, that is, does it induce a non-empty face?) We have a necessary condition from the results of Section 3. Let $T$ 
be a (degenerate) tooth meeting three handles. If there exists a $c$-tight factor, then by Lemma 3.4, there is a one, $x$, that saturates $T$. Therefore, there is a handle $H$ meeting $T$ for which $x(H \cap T: T \backslash H) \geq 2$. We will say in this case that $T$ occupies $H$ (with respect to $x$ ). It follows from Theorem 3.6 that no other tooth can occupy $H$. Therefore, the number of teeth meeting three handles cannot exceed the number of handles. (As an example of a 3-bipartition inequality that cannot be supporting because it violates this condition, consider the one having three handles of size five, and five teeth of size three, such that each tooth intersects each handle in a single node.) More generally, there must be an injection from the set of such teeth to the set of all handles so that each such tooth is mapped to a handle that it meets. This condition can be described in terms of the existence of a matching in a bipartite graph, where there is a node for each handle and a node for each tooth meeting three handles, and adjacency corresponds to non-empty intersection.

The above necessary condition for a bipartition inequality to be supporting is almost sufficient, but it needs to be amended to handle some exceptions. To give one example of such an exception, consider the bipartition inequality having three handles of size three and three teeth of size three, such that each tooth meets each handle in exactly one node, and there are ten nodes in total. Here we see that the matching condition is satisfied; in fact, if there were nine nodes only, the inequality would be supporting. However, there must be a subtour through the node that is in no handle, and this makes it impossible to obtain a tight factor. Notice that this difficulty persists if there are one, two, or three nodes not in any handle, but disappears if there are four or more. We can deal with the exceptions by modifying the definition of the bipartite graph mentioned above.

Given the bipartition inequality $c x \leq c_{0}$, let $n_{T}$ denote the number of pendent teeth, and let $n_{0}$ denote the number of isolated nodes, that is, nodes in no handle or tooth, let $V_{H}$ denote $\left\{H_{i}: 1 \leq i \leq h\right\}$, let

$$
S= \begin{cases}\left\{v, v^{\prime}\right\}, & \text { if } n_{T}=0 \text { and }\left|V_{H}\right| \geq 2 \text { with } 1 \leq n_{0} \leq 3 \\ \{v\}, & \text { if } n_{T}=1 \text { and }\left|V_{H}\right| \geq 2 \\ \emptyset, & \text { otherwise, }\end{cases}
$$

and let $V_{T}$ denote $\left\{T_{j}: d_{j}=3\right\} \cup S$. We define the graph $G(c)$ to have nodeset $V_{H} \cup V_{T}$, with node $H_{i}$ adjacent to a node $T_{j}$ if and only if $H_{i} \cap T_{j} \neq \emptyset$ and every node of $S$ 
adjacent to every handle node $H_{i}$. (There are no other adjacencies.) Note that $G(c)$ is bipartite with bipartition $\left\{V_{H}, V_{T}\right\}$. In particular, no matching of $G(c)$ can have cardinality larger than $V_{T}$. Whether this bound is tight or not determines whether the inequality is supporting. (We remark that it is easy to check that every bipartition inequality having fewer than two handles is supporting.)

Theorem 5.2 Let $c x \leq c_{0}$ be a 3-bipartition inequality having at least two handles. Then $c x \leq c_{0}$ is supporting for $P^{3}$ if and only if there exists a matching in $G(c)$ of cardinality $\left|V_{T}\right|$.

A 3-bipartition inequality is not dominated by a non-negativity inequality if and only if for every edge, there is a tight factor using the edge. Therefore, it should not be too surprising that a further refinement of the matching approach allows us to characterize such inequalities. We state this result next. (Notice that it does provide the promised good characterization, and hence shows that Theorem 5.1 is also a good characterization.) In fact, we will not actually prove Theorem 5.2, since we do not need it, and its proof is similar to the proof of Theorem 5.3. (Again, it is easy to check that a bipartition inequality having fewer than two handles cannot be dominated by a non-negativity inequality.)

Theorem 5.3 The 3-bipartition inequality $c x \leq c_{0}$ is not dominated by a non-negativity inequality if and only if there exists a matching of cardinality $\left|V_{T}\right|$ in $G(c) \backslash\left\{H_{i}, H_{l}\right\}$ for every pair of nodes $H_{i}, H_{l}$ in $V_{H}$.

The proof of this theorem requires some technical ideas that will also be useful later. A node $v$ is a tip of $c x \leq c_{0}$ if it is in a tooth but in no handle. A factor $x^{*}$ is said to strongly saturate a tooth $T_{j}$ of $c x \leq c_{0}$ if it saturates $T_{j}$ and, if $d_{j}=2, T_{j}=\{p, q, r\}$, and $q$ is a tip, then $x_{p q}^{*}=x_{q r}^{*}=1$. A factor is special if it is c-tight, simple, and strongly saturates every tooth.

Lemma 5.4 Let $c x \leq c_{0}$ be a supporting 3-bipartition inequality, and let $x^{*}$ be a c-tight simple factor. Let $\tilde{E}=\left\{e \in E: c_{e}=0\right.$ and $e$ is not incident to any tip $\}$. Then there exists a special factor $\tilde{x}$ such that for all $e \in \tilde{E}, \tilde{x}_{e}=1$ whenever $x_{e}^{*}=1$. 
Proof: Let $x^{*}$ be a $c$-tight simple factor. Suppose that there exists a tooth $T$ not strongly saturated by $x^{*}$. Let $d$ be the number of handles intersected by $T$. We demonstrate below how a new $c$-tight simple factor $\hat{x}$ can be constructed from $x^{*}$ such that the new factor satisfies $\hat{x}_{e}=1$ for all $e \in \tilde{E}$ with $x_{e}^{*}=1$ and strongly saturates $T$ as well as all teeth that are strongly saturated by $x^{*}$. By repeating this process, we can construct $\tilde{x}$, as required.

We distinguish four cases:

Case 1. $d=1$. Then there exists a tip $u \in T$ such that $x^{*}$ contains $e=u v$ with $c_{e}=0$. Applying 2OPT with respect to $T$, we obtain $\hat{x}$ from $x^{*}$ by replacing $e$ and another edge $e^{\prime} \in E(H)$ (since otherwise $c_{e^{\prime}}>0$ implies $c \hat{x}>c_{0}$ ), where $H$ intersects $T$, with an edge in $E\left(T_{j}\right)$ and some other edge, as required.

Case 2. $d=2$. Let $r \in H_{1} \cap T$ and $r^{\prime} \in H_{2} \cap T$. If $T$ is degenerate, then $c_{r r^{\prime}}=2$. Applying 2OPT with respect to $T$, we obtain $\hat{x}$ from $x^{*}$ by replacing some edges $r v$ and $r^{\prime} v^{\prime}$ in $x^{*}$ with $r r^{\prime}$ and $v v^{\prime}$. Since $c \hat{x} \leq c_{0}$, we must have $c_{r v}=c_{r v^{\prime}}=1$ and $c_{v v^{\prime}}=0$, and the required $\hat{x}$ is obtained.

Now consider $T=\left\{r, w, r^{\prime}\right\}$ with a tip $w$, and consider the subcases:

Case 2a. If $x^{*}$ satisfies $x_{r r^{\prime}}^{*}=x_{r w}^{*}=1$, then we have some $q \notin T$ with $x_{w q}^{*}=1$, and replacing $r r^{\prime}$ and $w q$ with $w r^{\prime}$ and $q r$ yields the desired $\hat{x}$.

Case 2b. If $x_{r r^{\prime}}^{*}=1$ and $x_{r w}^{*}=x_{w r^{\prime}}^{*}=0$, then applying 2OPT results in a factor violating $c x \leq c_{0}$, a contradiction.

Case 2c. If $x_{r w}^{*}=1$ and $x_{r r^{\prime}}^{*}=x_{w r^{\prime}}^{*}=0$, then applying 2OPT with respect to $T$ gives the desired $\hat{x}$.

Case 2d. If $x_{r w}^{*}=x_{r r^{\prime}}^{*}=x_{w r^{\prime}}^{*}=0$, then applying 2OPT with respect to $T$ yields a new factor. Set the new factor to be $x^{*}$, and we are in the Case $2 \mathrm{c}$.

Case 3. $d=3$. Let $r_{i} \in H_{i} \cap T, i=1,2,3$. So by Theorem $3.6 x_{e}^{*}=0$ for $e \in E(T)$.

First, let $C_{i}$ be the subtour in $x^{*}$ that contains $v_{i} r_{i}$ and $r_{i} s_{i}$, and $2 k_{i}+1$ be the number of teeth intersecting $H_{i}$. By Theorem 3.7, at least one of $c_{v_{i} r_{i}}$ and $c_{r_{i} s_{i}}$ is positive. Further, since $x^{*}$ is simple, either $c_{v_{i} r_{i}}=0$ or $c_{r_{i} s_{i}}=0$ implies by (a) of Theorem 3.7 that $\left|\left\{e \in \delta(H): x_{e}^{*}>0<c_{e}\right\}\right|=2 k_{i}+1$, which is impossible, since $x^{*}(T)=0$. So $v_{i}, s_{i} \in H_{i}$.

Next, let $v^{\prime} v_{i}, s^{\prime} s_{i} \in C_{i}$. Here we may assume without loss of generality that there exist no four distinct nodes $u, v, s, r$ in any handle $H$ such that $c_{u v}=c_{s r}=1$, and $u v$ and $s r$ belong to different subtours in $x^{*}$. (If this is the case, then replace $u v$ and 
sr by us and vs, and call the new factor $x^{*}$.) So all edges in $E\left(H_{i}\right)$ covered by $x^{*}$ belong to $C_{i}$. Notice that if $\left|C_{i}\right|=4$, then $v^{\prime}=s^{\prime}$, and by Theorem 3.7, all edges of $C_{i}$ are in $E\left(H_{i}\right)$ and $C_{i}$ contains no nodes in any tooth except $r_{i}$. It follows that $\left|C_{i}\right|=x^{*}\left(H_{i}\right)=\left|H_{i}\right|-k_{i} \geq 2 k_{i}+1+3-k_{i} \geq 5$, a contradiction.

Since $\left|C_{i}\right| \geq 5$ for $i=1,2,3$, the required $\tilde{x}$ can be constructed by replacing $v_{1} r_{1}, r_{1} s_{1}$, $v_{2} r_{2}, r_{2} s_{2}, v_{3} r_{3}$ with $v_{1} s_{1}, v_{2} s_{2}, r_{1} r_{2}, r_{2} r_{3}, r_{1} v_{3}$.

The proof is complete.

We are now able to prove that the matching condition of Theorem 5.3 is necessary.

Proof of necessity in Theorem 5.3. Let $H_{i}$ and $H_{l}$ be handles. Let $v_{i} \in H_{i} \cap T_{j}$ and $v_{l} \in H_{l} \cap T_{p}$ be a pair of nodes satisfying $c_{v_{i} v_{l}}=0$. Since $c x \leq c_{0}$ is not dominated by a non-negativity inequality, there exists a $c$-tight factor $x^{*}$ such that $x_{v_{i} v_{l}}^{*}=1$. Moreover, since $v_{i} v_{l} \in \tilde{E}$, by Lemma 5.4, there exists a special factor $\tilde{x}$ with $\tilde{x}_{v_{i} v_{l}}=1$. This implies that no degenerate tooth can occupy $H_{i}$ or $H_{l}$ and subtour $C_{0}$ of $\tilde{x}$ containing any isolated node or a tip cannot contain a node in $H_{i}$ or $H_{l}$. (Otherwise by Theorem 3.7, $\tilde{x}$ is not $c$-tight.) Now we construct a matching $M$ in $G(c) \backslash\left\{H_{i}, H_{l}\right\}$.

We begin with $M=\emptyset$. For every degenerate tooth $T_{j}$ that occupies a handle $H_{i}$ in $\tilde{x}$, we put the corresponding edge $\left(T_{j}, H_{i}\right)$ into $M$. As observed above, $M$ remains a matching. If $S=\emptyset$, we are done. So we just have to handle the two special cases.

Case 1. $n_{T}=0$ and $\left|V_{H}\right| \geq 2$ with $1 \leq n_{0} \leq 3$.

Let $C_{0}$ be a subtour of $\tilde{x}$ containing at least one isolated node. Since $n_{0} \leq 3, \tilde{x}$ is special, and $n_{T}=0, C_{0}$ must contain at least one node from some handle $H_{i}$. we have $c_{e}=0$ for every edge e of $C_{0}$ in $\delta\left(H_{i}\right)$. If $H_{i}$ is the only handle having a node in $C_{0}$, then $\tilde{x}$ uses two edges in $\delta\left(H_{i}\right)$ for which $c_{e}=0$, and by Theorem 3.7, $\tilde{x}$ is not $c$-tight, a contradiction. So $C_{0}$ visits two handles, say $H_{1}, H_{2}$, that are not occupied by any degenerate tooth $T_{j}$. (For otherwise we can show by Theorem 3.7 that $\tilde{x}$ is not $c$-tight.) We add the two edges $\left(v, H_{1}\right),\left(v^{\prime}, H_{2}\right)$ to $M$, where $\left\{v, v^{\prime}\right\}=S$.

Case 2. $n_{T}=1$ and $\left|V_{H}\right| \geq 2$.

Let $T_{1}=\left\{v_{1}, t_{1}\right\}$ be the pendent tooth with tip $t_{1}$ and $v_{1} \in H_{i}$. Let $C_{0}$ be the subtour of $\tilde{C}$ containing $v_{1} t_{1}$. Clearly, $C_{0}$ has to meet one handle $H_{l}$, possibly $l=i$, that is not occupied by any other degenerate tooth. (Isolated nodes, if any, can also be visited by $C_{0}$. So we set $|S|=1$.) Add the edge $\left(v, H_{l}\right)$ to $M$, where $\{v\}=S$. 
The following construction, called the $C$-construction, will be useful in the sequel. Let $M$ be a matching of $G(c)$ of cardinality $\left|V_{T}\right|$. For each tooth $T_{j}$, construct a path $P_{j}$ of length $\left|T_{j}\right|-1$ in $T_{j}$ such that

(i) If $T_{j}$ intersects a handle $H_{i}$ in two nodes $r, s$, then $r s$ is an edge of $P_{j}$;

(ii) $P_{j}$ enters and leaves a handle $H_{i}$ if and only if $H_{i} T_{j} \in M$.

Then for each handle $H_{i}$, there are $2 k_{i}+1$ paths of $G(V, C)$ visiting $H_{i}$, and at least $2 k_{i}$ of them have an end in $H_{i}$. Choose $2 k_{i}$ such nodes and $k_{i}$ paths $Q_{i \ell}$ joining them in pairs, so that every node of $H_{i}$ not in any $P_{j}$ is in exactly one of these paths. Define the set $C$ to be the union of the edge sets of all of the $P_{j}$ and all of the $Q_{i \ell}$. Note that $G(V, C)$ has no vertex of degree more than two, it has a node of degree 1 in each handle not covered by $M$, and $\left|C \cap E\left(H_{i}\right)\right|=\left|H_{i}\right|-k_{i}-1$ for each handle $H_{i}$. Also,

$$
\begin{aligned}
c(C) & =\sum_{i=1}^{h}\left(\left|H_{i}\right|-k_{i}-1\right)+\sum_{j=1}^{t}\left(\left|T_{j}\right|-1\right)+\sum_{j=t+1}^{t+m} \frac{d_{j}}{d_{j}-1}\left(\left|T_{j}\right|-1\right) \\
& =\sum_{i=1}^{h}\left(\left|H_{i}\right|-k_{i}-1\right)+\sum_{j=1}^{t+m} d_{j}+\sum_{j=1}^{t}\left(\left|T_{j}\right|-d_{j}-1\right)+\sum_{j=t+1}^{t+m} \frac{d_{j}}{d_{j}-1}\left(\left|T_{j}\right|-d_{j}\right) \\
& =\sum_{i=1}^{h}\left|H_{i}\right|+\sum_{i=1}^{h} k_{i}+\sum_{j=1}^{t}\left(\left|T_{j}\right|-d_{j}-1\right)+\sum_{j=t+1}^{t+m} \frac{d_{j}}{d_{j}-1}\left(\left|T_{j}\right|-d_{j}\right) .
\end{aligned}
$$

This construction allows us to construct (many) special factors. In particular, if $G(c) \backslash\left\{H_{p}, H_{q}\right\}$ has a matching of cardinality $\left|V_{T}\right|$, then $G(V, C)$ will have a node of degree 1 in each of $H_{p}, H_{q}$. This allows us to add edges to $C$ to form a special factor. Besides the flexibility in the choice of $p, q$, there may be flexibility in the choice of the paths $P_{j}$, of the $2 k_{i}$ nodes in $H_{i}$ for each $i$, and of the $Q_{i \ell}$. We use this construction repeatedly to prove both Theorem 5.3 and Theorem 5.1 .

Proof of Theorem 5.3. We have already proved the necessity of the matching condition. For sufficiency, we need to show that for any edge $u v$, there exists a tight factor using $u v$. For many choices of $u v$, this is easy.

If $u v \in E\left(H_{i}\right)$ for some $i$, we first choose $M$ so that $H_{i}$ is not covered. Then it is easy to ensure that $u v$ is an edge of some $P_{j}$ or some $Q_{i \ell}$.

If $u v \in E\left(T_{j}\right)$ for some $j$, then it is easy to ensure that $u v \in P_{j}$, with two exceptions. In the first exceptional case, $d_{j}=2$. Say that $T_{j}=\{u, v, w\}$, and $H_{i}$ is the handle 
containing $v$. We choose $M$ so that $H_{i}$ is not covered. Then $P_{j}$ will use $u v$. In the second exceptional case, $T_{j}=\{u, v, w\}$, with $w$ a tip, $u \in H_{i}$ and $v \in H_{m}$. Then we choose $M$ so that $H_{i}$ is not covered, and choose the $Q_{i \ell}$ so that $u$ has degree one in $G(V, C)$. Then the special factor resulting contains $v w, w u$ and an edge $u s$ such that $c_{u s}=0$. Replacing $v w$ and $u s$ by $u v$ and $w s$ gives the required tight factor. (It is not special.)

If $c_{u v}=0$ it is easy in most cases to construct $C$ so that $u, v$ both have degree one or zero in $G(V, C)$. We treat only the cases where it is not. One case is where $u$, say, is a node of some handle $H_{i}$ but of no tooth. We choose $M$ so that $H_{i}$ is not covered, and construct $C$. Then $u$ is incident with two edges su, ur of $C$ and there is a $w \in H_{i}$ incident to just one edge of $C$. We replace $s u$, ur in $C$ by $s r, w u$. The other case is where $u$, say, is a tip of a tooth $T_{j}=\{u, a, b\}$ with $a \in H_{i}$ and $b \in H_{m}$. We choose $M$ so that $H_{m}$ is not covered, and choose the $Q_{i \ell}$ so that $b$ has degree one in $G(V, C)$. Then we replace $u b$ by $a b$ in $C$. Notice that we can apply these last techniques independently for either of $u$ or $v$, since we can choose $M$ to miss any two handles.

We are now ready to prove the main result of this section, Theorem 5.1. Since the necessity of the conditions follows from Theorem 4.1, Lemma 4.4, and Theorem 5.3, we need to prove sufficiency. We consider only the case where the number $h$ of handles defining $c x \leq c_{0}$ is more than 1 , for otherwise $c x \leq c_{0}$ is an SE constraint or a comb inequality, and hence the theorem holds by the well known polyhedral results for the travelling salesman polytope $P^{n}$. Since $c x \leq c_{0}$ is not dominated by a non-negativity inequality, by Theorem 5.3, there exists a matching of cardinality $\left|V_{T}\right|$ missing any two handles $H_{p}, H_{q}$. We will use these matchings and the $C$-construction to obtain tight factors containing specific edges.

Since there are at least two handles and no degenerate cut, there exist handles $H$ and $H^{\prime}$ and a tooth $T$ such that $T=\left\{r, w, r^{\prime}\right\}, T \cap H=\{r\}$, and $T \cap H^{\prime}=\left\{r^{\prime}\right\}$. Let $T^{\prime}$ be a tooth different from $T$ that intersects $H^{\prime}$, and let $s^{\prime} \in H^{\prime} \cap T^{\prime}$. Note that $T^{\prime}$ may intersect $H$. Let $T^{\prime \prime}$ be a tooth different from $T$ that intersects $H$. Figure 4 gives a picture of the situation, but it is not completely general. Choose a node $s \in H \cap T^{\prime \prime}$. We define $B$ to be $\left\{r s^{\prime}, r^{\prime} s, s s^{\prime}\right\} \cup\left\{w v: v \in V \backslash\left\{r, r^{\prime}\right\}\right\}$. Thus, $B$ forms a basis and $c_{e}=0$ for all $e \in B$. Let $f x \leq f_{0}$ be a facet-inducing inequality that dominates $c x \leq c_{0}$ with $f_{e}=0$ for all $e \in B$. Let $\alpha=f_{r r}$. We successively derive all values of $f_{e}$ by comparing tight factors, that is, 


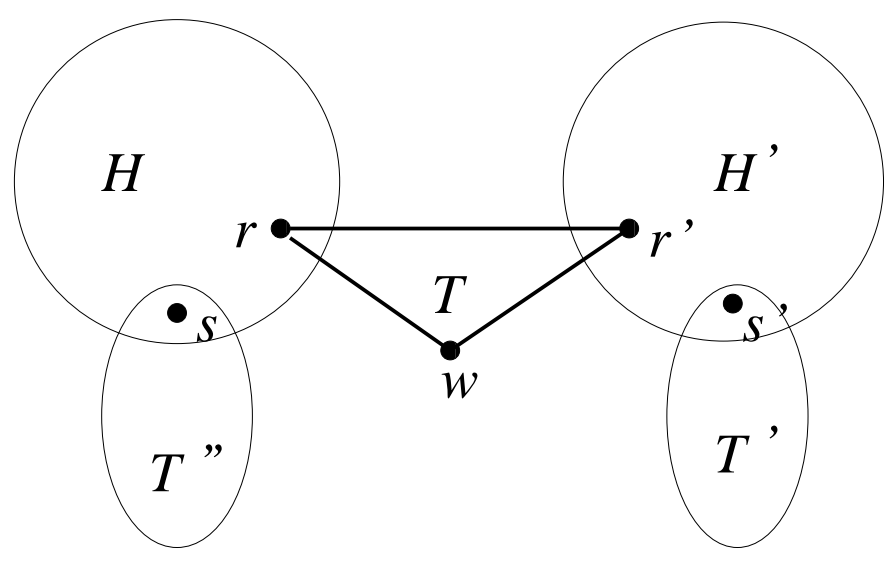

Figure 4: Proving sufficiency.

given tight factors $x, x^{\prime}$, using the fact that $0=c x-c x^{\prime}=f x-f x^{\prime}$ to derive values for the $f_{e}$. For a factor $x, x \triangle\left(e_{1}, \ldots, e_{l} ; e_{1}^{\prime}, \ldots, e_{l}^{\prime}\right)$ denotes the factor obtained from $x$ by replacing edges $e_{1}, \ldots, e_{l}$ with $e_{1}^{\prime}, \ldots, e_{l}^{\prime}$. (That is, we take the symmetric difference of the factor $x$ and the edge set $\left\{e_{1}, \ldots, e_{l}, e_{1}^{\prime}, \ldots, e_{l}^{\prime}\right\}$, but our notation makes explicit which edges are in and not in $x$.)

Claim 5.5 $f_{e}=\alpha$ for all $e \in E(T)$.

Proof: It is easy to arrange in the $C$-construction for $C$ to contain $r w$ and $w r^{\prime}$ and for $r$ and $s^{\prime}$ to be incident with exactly one edge of $C$. Therefore, there is a tight factor $x$ using $r w, w r^{\prime}$, and $r s^{\prime}$. Comparing $x$ with the tight factor $x \triangle\left(r s^{\prime}, w r^{\prime} ; r r^{\prime}, w s^{\prime}\right)$ gives $f_{w r^{\prime}}=\alpha$. Similarly, we have $f_{w r}=\alpha$, and $f_{r r^{\prime}}=\alpha$ by definition.

Claim 5.6 $f_{e}=0$ for all $e \in \delta(T)$ with $c_{e}=0$.

Proof: For any node $u$ such that $c_{u r}=0$, it is easy to arrange in the $C$-construction for $C$ to contain $r w$ and $w r^{\prime}$, and for $u$ and $r$ to be incident with at most one edge from $C$. Thus there is a tight factor $x$ using $r w, w r^{\prime}$, and $u r$. Comparing $x$ with the tight factor $x \triangle\left(r u, w r^{\prime} ; r r^{\prime}, w u\right)$ gives $f_{r u}=0$. Similarly, for each $u$ such that $c_{r^{\prime} u}=0$, we have $f_{r^{\prime} u}=0$. Finally, for every $u$ such that $c_{w u}=0$, we have $f_{w u}=0$ by definition.

Claim 5.7 If $e \in E(H) \cup E\left(H^{\prime}\right)$ with $c_{e}=1$, then $f_{e}=\alpha$. 
Proof: As before, we can construct a tight factor $x$ using $s s^{\prime}, r w, w r^{\prime}$. Notice that $x^{\prime}=$ $x \triangle\left(s s^{\prime}, r w ; w s^{\prime}, r s\right)$ is also a factor, since by Theorem $3.7, x$ contains edges $s u \in E\left(T^{\prime \prime}\right)$ and $s^{\prime} u^{\prime} \in E\left(T^{\prime}\right)$, but neither $u r$ nor $s^{\prime} r^{\prime}$. So, comparing $x$ and $x^{\prime}$ yields $f_{r s}=\alpha$.

Next, consider any pair of nodes $u, v \in H \backslash\{r\}$. We can construct a tight factor $x$ using $u v, r s^{\prime}, r w, w r^{\prime}$. (The $C$-construction will automatically use the last two edges, and we can arrange that $u v$ is in some $Q_{i \ell}$, and that $r$ and $s^{\prime}$ be incident to exactly one edge of $C$.) Observe that $x^{\prime}=x \triangle\left(u v, w r, r s^{\prime} ; u r, v r, w s^{\prime}\right)$ and $x^{\prime \prime}=x \triangle\left(u v, r s^{\prime}, w r^{\prime} ; v r, w u, r^{\prime} s^{\prime}\right)$ are also tight factors, and thus comparing $x^{\prime}$ and $x^{\prime \prime}$ yields $f_{u r}=f_{r^{\prime} s^{\prime}}$. So we can derive that $f_{r^{\prime} s^{\prime}}=f_{u r}=f_{r s}=\alpha$ for all $u \in H \backslash\{r\}$. Now comparing $x$ and $x^{\prime}$ gives $f_{u v}=\alpha$. It follows that $f_{e}=\alpha$ for all $e \in E(H)$ with $c_{e}=1$. By symmetry, $f_{e}=\alpha$ for all $e \in E\left(H^{\prime}\right)$ with $c_{e}=1$.

Claim 5.8 If $e \in \delta(H) \cup \delta\left(H^{\prime}\right)$ with $c_{e}=0$, then $f_{e}=0$.

Proof: By Claim 5.6 and the symmetry between $H$ and $H^{\prime}$, we need only consider $e=u v$ with $v \in H$ and $\{u, v\} \cap T=\emptyset$. Suppose that there exists a tight factor $x$ that uses $u v, q r, r w, w r^{\prime}$ for some $q \in H$, and moreover, uses edges $q q^{\prime}$ and $v v^{\prime}$, where $q^{\prime}, v^{\prime}$ are distinct and different from $q, v, r$. Then $x \triangle(u v, q r ; q v, u r)$ is also a tight factor, and by Claims 5.5, 5.6, and 5.7, comparing it with $x$ shows $f_{u v}=0$. Now we explain how to construct $x$.

If $v$ is contained in some other tooth, then by Theorem 3.7, a tight factor $x$ containing $u v, r w, w r^{\prime}$ satisfies the desired property and such an $x$ is easily constructed using the $C$-construction.

If $v$ is in no tooth, let $x^{\prime}$ be a special factor containing $u u^{\prime}$ for some $u^{\prime} \in H \cap \hat{T}$ with $c_{u u^{\prime}}=0$. Then let $v \ldots v_{l}$ be a path of all nodes in $H$ that are not covered by any tooth $\left(v_{l}\right.$ may be $v$ itself), and the required $x$ can be contructed from $x^{\prime}$ by inserting $v \ldots v_{l}$ between $u u^{\prime}$ in $x^{\prime}$ and removing those nodes from other positions in $x^{\prime}$.

Now suppose that $T^{\prime}$ is intersected by another handle $H^{\prime \prime}$ with $\left\{s^{\prime \prime}\right\}=T^{\prime} \cap H^{\prime \prime}$ and $w^{\prime}$ is a tip in $T^{\prime}$.

Claim 5.9 There exists some scalar $\alpha^{\prime}$ such that $f_{e}=\alpha^{\prime}$ for $e \in E\left(T^{\prime}\right)$; 
Proof: Let $\alpha^{\prime}=f_{s^{\prime} s^{\prime \prime}}$. By the $C$-construction, we obtain a special factor $x$ containing $r s^{\prime}, s^{\prime} w^{\prime}, w^{\prime} s^{\prime \prime}$. Comparing $x$ with $x \triangle\left(r s^{\prime}, w^{\prime} s^{\prime \prime} ; r w^{\prime}, s^{\prime} s^{\prime \prime}\right)$ implies by Claim $5.6 f_{w^{\prime} s^{\prime \prime}}=$ $\alpha^{\prime}$. Similarly, let $x$ be a special factor containing $r^{\prime} s^{\prime \prime}, s^{\prime} w^{\prime}, w^{\prime} s^{\prime \prime}$; comparing $x$ with $x \triangle\left(r^{\prime} s^{\prime \prime}, w^{\prime} s^{\prime} ; r^{\prime} w^{\prime}, s^{\prime} s^{\prime \prime}\right)$ implies $f_{w^{\prime} s^{\prime}}=\alpha^{\prime}$.

Claim $5.10 f_{e}=0$ for all $e \in \delta\left(T^{\prime}\right)$ with $c_{e}=0$.

Proof: By Claims 5.5-5.8 we only need to consider edges $e=u v$ with $c_{w v}=0, v \in$ $\left\{w^{\prime}, s^{\prime \prime}\right\}$ and $u \notin T \cup H \cup H^{\prime}$. Let $x$ be a special factor containing $u s^{\prime}, s^{\prime} w^{\prime}, w^{\prime} s^{\prime \prime}$. Using Claim 5.9, comparing $x$ with $x \triangle\left(u s^{\prime}, w^{\prime} s^{\prime \prime} ; u w^{\prime}, s^{\prime} s^{\prime \prime}\right)$ yields $f_{u w^{\prime}}=f_{u s^{\prime}}=0$. Now consider $u s^{\prime \prime}$. Since $c_{u s^{\prime \prime}}=0, u \notin H^{\prime \prime}$. Let $x^{\prime}$ be a special factor containing $u s^{\prime \prime}, s^{\prime \prime} w^{\prime}, w^{\prime} s^{\prime}$; comparing $x^{\prime}$ with $x^{\prime} \triangle\left(u s^{\prime \prime}, w^{\prime} s^{\prime} ; u w^{\prime}, s^{\prime} s^{\prime \prime}\right)$ shows $f_{u s^{\prime \prime}}=f_{u w^{\prime}}=0$.

Now, notice that $f_{e}$ 's for $e \in E\left(T^{\prime}\right) \cup \delta\left(T^{\prime}\right)$ are proportional to those for $e \in E(T) \cup$ $\delta(T)$, and so we can apply Claim 5.7 with respect to $T^{\prime}$. It follows that $\alpha=\alpha^{\prime}$. Since $c x \leq c_{0}$ has no degenerate cut, by repeated applications of Claims 5.6-5.10, we derive that for any handle $\hat{H}$ and any nondegenerate nonpendent tooth $\hat{T}$,

$f_{e}=\alpha, \forall e \in E(\hat{H}) \cup E(\hat{T})$ with $c_{e}=1$, and

$f_{e}=0, \forall e \in \delta(\hat{H}) \cup \delta(\hat{T})$ with $c_{e}=0$.

The above properties of $f$ are used implicitly in the sequel.

Let $\tilde{T}$ be any pendent tooth intersecting some handle $\tilde{H}$, and $u \in \tilde{T} \backslash H^{\prime}$.

Claim $5.11 f_{e}=\alpha c_{e}$ for $e \in E(\tilde{T})$.

Proof: We may assume without loss of generality that $H^{\prime}=\tilde{H}$. Suppose $\{v\}=\tilde{T} \cap H^{\prime}$. Then let $x$ be the special factor containing $v r, r w, w r^{\prime}$ and $r^{\prime} v^{\prime}$. Clearly, if $|T|=2, x$ contains $u v$. For $|\tilde{T}|=3$, let $q \in \tilde{T} \backslash H^{\prime}$, and we may assume that this $x$ contains $q u$, $u v$. Comparing $x$ with $x \triangle\left(v r, u v, r^{\prime} v^{\prime} ; u r, r^{\prime} v, v v^{\prime}\right)$ implies $f_{u v}=\alpha$. If $|\tilde{T}|=3$, by symmetry, we derive $f_{q v}=\alpha$. Then comparing $x$ with $x \triangle(v r, u q ; u r, v q)$ yields $f_{u q}=\alpha$.

Now suppose that $\{v, q\}=\tilde{T} \cap H^{\prime}$. First, let $x$ be a special factor containing $r v, r w, w r^{\prime}, v q, q u$ and $r^{\prime} v^{\prime} \in E\left(H^{\prime}\right)$. So comparing $x$ with $x \triangle\left(r v, q u, r^{\prime} v^{\prime} ; r u, r^{\prime} v, q v^{\prime}\right)$ implies $f_{u q}=\alpha$. By symmetry $f_{u v}=\alpha$. Next, observe that there exists a special factor $x^{\prime}$ containing $r^{\prime} u, r w, w r^{\prime}, v q, q u$, and $x^{\prime}$ must contain $v v^{\prime}$ for some $v^{\prime} \in H^{\prime}$. Comparing $x^{\prime}$ with $x^{\prime} \triangle\left(u r^{\prime}, v q ; u v, q r^{\prime}\right)$ implies $f_{v q}=2 \alpha$. 
Claim $5.12 f_{e}=0$ for $e=u u^{\prime}$ with $u^{\prime}$ in any pendent tooth different from $\tilde{T}$.

Proof: If $u^{\prime}$ is contained in a handle, then $f_{u u^{\prime}}=0$ by previous derivations. So suppose that $u^{\prime}$ is contained in no handle. If $\hat{T}$ is the tooth containing $u^{\prime}$, then there exists a special factor $x^{\prime}$ containing $u^{\prime} v, u^{\prime} u^{\prime \prime}, r^{\prime} w, w r, v^{\prime} r^{\prime}$, where $u^{\prime} u^{\prime \prime} \in E(\hat{T}), v \in H^{\prime} \cap \tilde{T}$ and $v^{\prime} \in H^{\prime}$. If $x$ contains $u u^{\prime \prime}$, then comparing $x$ with $x \triangle\left(u^{\prime} v, u u^{\prime \prime} ; u u^{\prime}, v u^{\prime \prime}\right)$ shows $f_{u u^{\prime}}=0$. Otherwise we have two cases. First, if $\tilde{T} \cap H^{\prime}=\{v\}$, then assume that $x$ contains $u v$, and comparing $x$ with $x \triangle\left(u^{\prime} v, u v, r^{\prime} v^{\prime} ; u u^{\prime}, v r^{\prime}, v v^{\prime}\right)$ gives $f_{u u^{\prime}}=0$. Second, if $\tilde{T} \cap\{v, q\}$, we may assume that $x$ contains $v q, u q$, and then comparing $x$ with $x \triangle\left(u^{\prime} v, u q, r^{\prime} v^{\prime} ; u u^{\prime}, v r^{\prime}, q v^{\prime}\right)$ yields $f_{u u^{\prime}}=0$.

Claim 5.13 For any degenerate tooth $\tilde{T}, f_{e}=\alpha c_{e}$ for all $e \in E(\tilde{T})$.

Proof: First, suppose that $\tilde{T}$ intersects two handles $H_{1}$ and $H_{2}$ with $u_{i} \in H_{i} \cap \tilde{T}$, $i=1,2$. Assume without loss of generality that $H=H_{1}$ since each handle intersects a nondegenerate tooth that connects two handles. There exists a special factor $x$ containing $r u_{2}, r w, w r^{\prime}$, and some edge $u^{\prime} v^{\prime} \in E\left(H_{2}\right)$. For $|\tilde{T}|=2$, comparing $x$ with $x \triangle\left(r u_{2}, u^{\prime} v^{\prime}, u_{1} u_{2} ; r u_{1}, u^{\prime} u_{2}, v^{\prime} u_{2}\right)$ yields $f_{u_{1} u_{2}}=2 \alpha$. For $|\tilde{T}|=3$, we may assume without loss of generality that $\left\{u_{2}, q\right\}=\tilde{T} \cap H_{2}$. The factor $x$ then contains $u_{2} q, q u_{1}$. Comparing $x$ and $x \triangle\left(r u_{2}, q u_{1}, u^{\prime} v^{\prime} ; r u_{1}, u^{\prime} u_{2}, v^{\prime} q\right)$ implies $f_{u_{1} q}=2 \alpha$. By symmetry, $f_{u_{1} u_{2}}=2 \alpha$. Finally, let $q^{\prime} \in H_{2} \cap \hat{T}$ with $\hat{T} \neq \hat{T}$, and $x^{\prime}$ be a special factor containing $u_{1} q^{\prime}$. So $x^{\prime}$ contains $u_{1} q, q u_{2}$. Comparing $x^{\prime}$ with $x^{\prime} \triangle\left(u_{1} q^{\prime}, u_{2} q ; u_{1} u_{2}, q q^{\prime}\right)$ yields $f_{q u_{2}}=3 \alpha$.

Second, suppose that $T$ intersects three handles, say $H_{1}, H_{2}, H_{3}$, and let $r_{i} \in H_{i} \cap \tilde{T}$, $i=1,2,3$. By the $C$-construction, there exists a special tight factor $x$ containing $v_{1} r_{2}, r_{2} r_{3}, r_{3} r_{1}, r_{1} s_{1}$ where $v_{1}, s_{1} \in H_{1}$, and $v_{1}$ and $s_{1}$ are each contained in teeth. Furthermore, there exist nodes $v_{2}, s_{2} \in H_{2}$ and $v_{3}, s_{3} \in H_{3}$ such that $x$ contains $v_{2} s_{2}$ and $v_{3} s_{3}$. Since $x$ is special, then $x^{*}=x \triangle\left(v_{1} r_{2}, r_{2} r_{3}, r_{3} r_{1}, v_{2} s_{2}, v_{3} s_{3} ; v_{1} r_{1}, v_{2} r_{2}, r_{2} s_{2}, v_{3} r_{3}, r_{3} s_{3}\right)$ is a tight factor. Comparing $x$ with $x^{*}$ implies $f_{r_{1} r_{3}}+f_{r_{2} r_{3}}=3 \alpha$. By symmetry, we have $f_{r_{1} r_{2}}+f_{r_{2} r_{3}}=f_{r_{2} r_{1}}+f_{r_{3} r_{1}}=3 \alpha$. It follows that $f_{e}=\frac{3}{2} \alpha$ for $e \in E(\tilde{T})$.

Let $V_{0}$ be the collection of all nodes not contained in any handle or tooth, and $T_{0}$ be the collection of all nodes contained in some pendent tooth but in no handle.

Claim 5.14 $f_{e}=0$ for all $e \in E\left(V_{0}\right) \cup E\left(V_{0}: T_{0}\right)$. 
Proof: Let $x$ be a special factor containing $r s^{\prime}$. (Recall $r \in H \cap T, s^{\prime} \in H^{\prime} \cap T^{\prime}$.) For any $u v \in E\left(V_{0}\right)$, if $x$ contains $u v$, let $x^{*}=x$. Otherwise let $x^{*}$ be obtained from $x$ by applying 2OPT with respect to $S=\{u, v\}$. So $x^{*}$ is a special factor containing $r s^{\prime}$, $u v$. Now observe that either $x^{*} \triangle\left(u v, r s^{\prime} ; u r, v s^{\prime}\right)$ or $x^{*} \triangle\left(u v, r s^{\prime} ; u s^{\prime}, v r\right)$ is a tight factor, and comparing the resulting factor with $x^{*}$ yields $f_{u v}=0$.

Next, consider any $u \in V_{0}$ and $v \in T_{0}$. Assume that $v$ is contained in pendent tooth $\hat{T}$, and $v^{\prime} \in \hat{T} \cap H^{\prime}$. We distinguish the following two cases:

CASE 1: If $\hat{T}$ is the only pendent tooth, let $x$ be a special factor containing $v r^{\prime}, r^{\prime} w, w r$ and $v v^{\prime}$ with $v^{\prime} \in H^{\prime} \cap \hat{T}$. Thus we may assume that $x$ contains some $u u^{\prime}$ with $u^{\prime}$ in some handle, and hence $f_{u u^{\prime}}=0$. Further, note that $x \triangle\left(u u^{\prime}, v r^{\prime}, v v^{\prime} ; v^{\prime} r^{\prime}, u v, v u^{\prime}\right)$ is a tight factor, and so comparing it with $x$ implies $f_{w w}=0$.

CASE 2: Let $T^{\prime \prime}$ be another pendent tooth, and $v^{\prime \prime} \in T^{\prime \prime} \cap H^{\prime \prime}$. Let $x$ be a special factor containing $v^{\prime} v^{\prime \prime}$. Set $x^{*}=x$ if $x$ contains $u v$. If not, let $x$ contain $v q$ with $c_{v q}=f_{v q}=0$, and replace $v q$ with some subpath containing $u$ to obtain $x^{*}$ containing $u v$. Now comparing $x^{*}$ with $x^{*} \triangle\left(u v, v^{\prime} v^{\prime \prime} ; u v^{\prime}, v v^{\prime \prime}\right)$ shows $f_{u v}=0$.

Combining the above lemmas, we have $f_{e}=\alpha c_{e}$ for all $e \in E$. It follows that $c x \leq c_{0}$ induces a facet.

\section{Another facet}

Since the problem of existence of a restricted factor is solvable in polynomial time when $k=3$, we may hope that the optimal restricted factor problem is solvable in this case. Hence, we may hope that one could find a complete description by linear inequalities for $P^{3}$. A natural first candidate for such a description is the set of all degree, nonnegativity, and 3-bipartition constraints. However, this list is not sufficient in general. Consider the inequality $c x \leq c_{0}$ indicated in Figure 5, where numbers on edges are coefficients, missing edges have coefficient zero and the right-hand side is 16 . Let us first explain where this inequality comes from. There is a 3-bipartition inequality $d x \leq d_{0}$ having the same support, having three handles, and four degenerate teeth, one of size three and the others of size two. This inequality has a degenerate cut, and so is not facet-inducing by Theorem 4.1. In fact, the proof of that result allows us to identify a 
comb inequality $p x \leq p_{0}$ inducing a facet that properly contains the face $F$ of $P 3(9)$ induced by $d x \leq d_{0}$. Of course, there must be other inequalities inducing faces properly containing $F$. One of them can be obtained as follows. Consider the inequality $q x \leq q_{0}$ defined to be $d x-\alpha p x \leq d_{0}-\alpha p_{0}$, where $\alpha$ is chosen as large as possible so that it is valid for $P^{3}(9)$. Then $q x \leq q_{0}$ is equivalent to the inequality of Figure 5 .

Proposition 6.1 The inequality $c x \leq c_{0}$ of Figure 5 is facet-inducing for $P^{3}(9)$, and is not equivalent to any non-negativity or bipartition inequality.

The proof of this result is elementary, but not particularly short or illuminating, so it is not included here. It is not at all clear to us what class of inequalities this one might belong to, so we have no conjecture as to a complete description for $P^{3}(n)$.

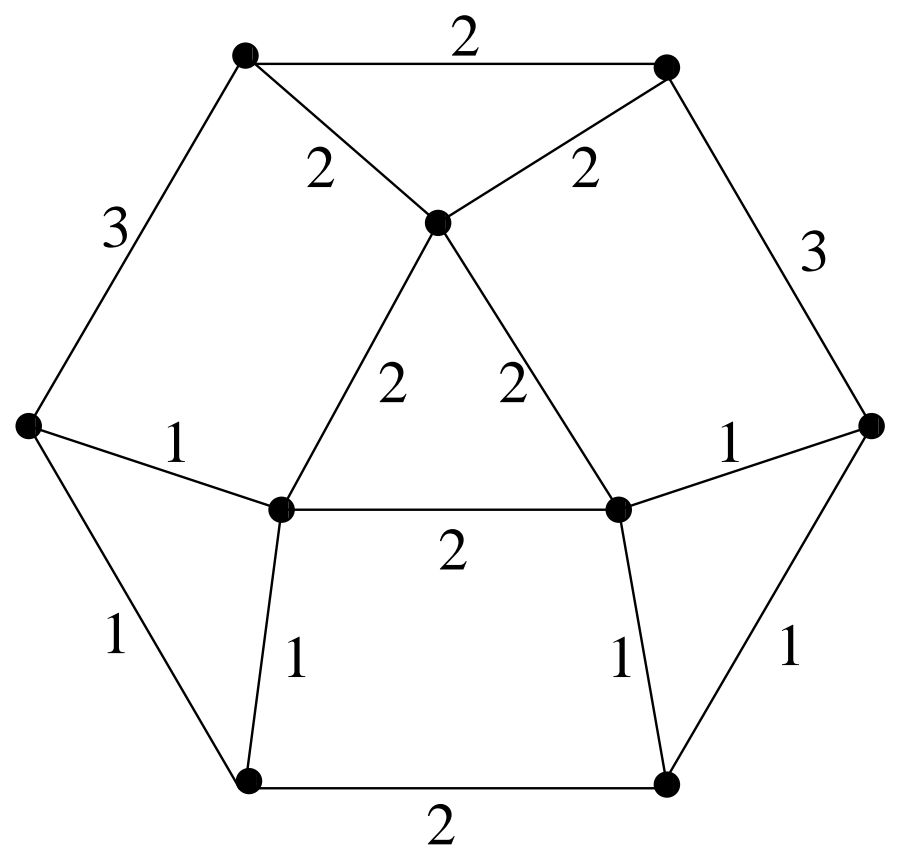

Figure 5: Another facet for $P^{3}$.

\section{References}

[1] S.C. Boyd and W.H. Cunningham, "Small travelling salesman polytopes," Mathematics of Operations Research 16 (1991) 259-271. 
[2] R.D. Carr, "Separating clique tree and bipartition inequalities in polynomial time", in: E. Balas and J. Clausen, eds. Integer Programming and Combinatorial Optimization, Lecture Notes in Computer Science 920, Springer, Berlin, 1995, pp. 40-49.

[3] G. Cornuéjols and W.R. Pulleyblank, "A matching problem with side conditions", Discrete Mathematics 29 (1980), 135-159.

[4] J. Edmonds, "Maximum matching and a polyhedron with 0,1-vertices," J. Res. Nat. Bur. Standards 69B, (1965) 147-153.

[5] M. Grötschel and M.W. Padberg, "Polyhedral theory," in: E. Lawler, J.K. Lenstra, A. Rinnooy Kan and D. Shmoys, eds., The Travelling Salesman Problem (Wiley, New York, 1985).

[6] M.X. Goemans and D.P. Williamson, "A general approximation technique for constrained forest problems", SIAM J. Computing 24, (1995), 296-317.

[7] M. Grötschel and M.W. Padberg, "On the symmetric travelling salesman problem II: lifting theorems and facets," Mathematical Programming 16 (1979) 281-302.

[8] M. Grötschel and W.R. Pulleyblank, "Clique tree inequalities and the symmetric travelling salesman problem," Mathematics of Operations Research 11 (1986) 537569.

[9] D.B. Hartvigsen, Extensions of Matching Theory, Ph.D. Thesis, Carnegie Mellon University, 1984.

[10] O. Vornberger, "Easy and hard cycle covers", preprint, Universität Paderborn, 1980 . 